\title{
Optimal bounds of restricted type for the Hardy operator minus the identity on the cone of radially decreasing functions
}

\author{
by \\ JAVIER SORIA (Barcelona)
}

\begin{abstract}
We find the norm of the Hardy operator minus the identity acting on the cone of radially decreasing functions on minimal Lorentz spaces (restricted type estimates).

The study of the Hardy operator (see (2)) on monotone functions has its origins in the works of Ariño-Muckenhoupt [1] and Sawyer [12, dealing with the characterization of the boundedness on the weighted Lorentz spaces $\Lambda^{p}(w)$ (see (11)), of the Hardy-Littlewood maximal operator, and the normability properties for these spaces, extending the well known results of Lorentz [11] (see [7, 6] for a modern account of this theory).

Recently, several results concerning the Hardy operator minus the identity have been considered (see [10, 5, 4]). In particular, in [10] the authors study the norm of this difference of operators, restricted to the cone of decreasing functions on $L^{p}\left(\mathbb{R}^{+}\right)$, and are able to calculate the exact constant for the case $p \in\{2,3,4, \ldots\}$.

In this paper we study the same kind of questions, but now we look at estimates of restricted type, and we will obtain a complete answer in the more general setting of rearrangement invariant (r.i.) spaces [3], and for the $n$-dimensional Hardy operator (see (1) for the definition and [9] for some previous results). The main tool we will use is the integral representation of Lemma 3 (see also [5]), which will allow us to easily prove the main estimate in Theorem 5 . This result introduces a new class of functions $R(X)$ (see Definition 6) which is identified, for the Lorentz space $L^{p, q}\left(\mathbb{R}^{n}\right)$, in Propositions 8 and 11. As a consequence, we find the best constants for the restricted type boundedness (see Propositions 9 and 12). Finally, in Theorem 13, we show that, in most cases, $R(X)$ is the minimal Lorentz space $\Lambda(X)$.
\end{abstract}

2010 Mathematics Subject Classification: 26D10, 46E30.

Key words and phrases: Hardy operator, restricted type, Lorentz spaces. 
We start by introducing some basic definitions and notations:

Definition 1. Let $X$ be an r.i. Banach function space in $\mathbb{R}^{n}$. We define $X_{\mathrm{rd}}=\{f \in X: f$ is a positive radially decreasing function $\}$; that is, $f(x)=\bar{f}(|x|) \in X$ and $\bar{f}$ is a positive decreasing function on $\mathbb{R}^{+}$.

The distribution function of $f$ is defined as

$$
\lambda_{f}(t)=\left|\left\{x \in \mathbb{R}^{n}:|f(x)|>t\right\}\right|,
$$

and the nonincreasing rearrangement of $f$ by

$$
f^{*}(t)=\inf \left\{s>0: \lambda_{f}(s) \leq t\right\} .
$$

The Hardy operator in $\mathbb{R}^{n}$ is

$$
S_{n} f(x)=\frac{1}{|B(0,|x|)|} \int_{B(0,|x|)} f(y) d y,
$$

and the Hardy operator in $\mathbb{R}^{+}$is

$$
S f(t)=\frac{1}{t} \int_{0}^{t} f(r) d r .
$$

REMARK 2. Observe that if $f(x)=\bar{f}(|x|)$, then

$$
\lambda_{f}(t)=v_{n} \lambda_{\bar{f}}^{n}(t),
$$

where $v_{n}$ is the measure of the unit ball of $\mathbb{R}^{n}$, and

$$
S_{n} f(x)=\frac{n}{|x|^{n-1}} S\left(r^{n-1} \bar{f}(r)\right)(|x|) .
$$

Also, if $f^{* *}(t)=t^{-1} \int_{0}^{t} f^{*}(s) d s$ is the maximal function of $f$ [3] then, for a radially (i.e., even) decreasing function $f(x)=\bar{f}(|x|)$ in $\mathbb{R}$, we have

$$
S_{1} f(x)-f(x)=S(\bar{f})(|x|)-\bar{f}(|x|)=f^{* *}(2|x|)-f^{*}(2|x|) .
$$

The next result is the key to obtaining the main estimate in Theorem 5 . It was first proved (for $n=1$ and with a more involved argument) in [5, Lemma 2.1(6)].

Lemma 3. If $f(x)=\bar{f}(|x|)$ is a positive radially decreasing function on $\mathbb{R}^{n}$, then

$$
S_{n} f(x)-f(x)=\frac{1}{|x|^{n}} \int_{\bar{f}(|x|)}^{\infty} \lambda_{\bar{f}}^{n}(t) d t, \quad x \in \mathbb{R}^{n} .
$$

Proof. For a fixed $x \in \mathbb{R}^{n}$ we consider the measure

$$
d \mu_{x}(y)=\frac{\chi_{B(0,|x|)}(y)}{|B(0,|x|)|} d y-\delta_{x}(y),
$$


which is positive on balls centered at the origin:

$$
\mu_{x}(B(0, a))= \begin{cases}(a /|x|)^{n}, & a \leq|x|, \\ 0, & a>|x| .\end{cases}
$$

Therefore, since for a positive radially decreasing function $f$, the level sets are balls centered at zero and of radius $\lambda_{\bar{f}}(t)$, we have

$$
\begin{aligned}
S_{n} f(x)-f(x) & =\int_{\mathbb{R}^{n}} f(y) d \mu_{x}(y)=\int_{0}^{\infty} \mu_{x}(f>t) d t \\
& =\int_{0}^{\infty} \mu_{x}\left(B\left(0, \lambda_{\bar{f}}(t)\right)\right) d t=\frac{1}{|x|^{n}} \int_{\bar{f}(|x|)}^{\infty} \lambda_{\bar{f}}^{n}(t) d t .
\end{aligned}
$$

REMARK 4. Observe that using (3) we also have

$$
S_{n} f(x)-f(x)=\frac{1}{v_{n}|x|^{n}} \int_{f(x)}^{\infty} \lambda_{f}(t) d t .
$$

Theorem 5. Let $X$ be an r.i. Banach function space in $\mathbb{R}^{n}$. Then

$$
\left\|S_{n} f-f\right\|_{X} \leq \int_{0}^{\infty} \lambda_{\bar{f}}^{n}(t)\left\|\frac{1}{\lambda_{\bar{f}}^{n}(t)+|\cdot|^{n}}\right\|_{X} d t
$$

for every $f \in X_{\mathrm{rd}}$. Moreover, the inequality is sharp.

Proof. Using Lemma 3 and Minkowski's integral inequality, we have

$$
\left\|S_{n} f-f\right\|_{X} \leq \int_{0}^{\infty} \lambda_{\bar{f}}^{n}(t)\left\|\frac{\chi_{(\bar{f}(\mid \cdot), \infty)}(t)}{|\cdot|^{n}}\right\|_{X} d t .
$$

Set now

$$
g_{t}(x)=\frac{1}{\lambda_{\bar{f}}^{n}(t)+|x|^{n}}, \quad h_{t}(x)=\frac{\chi_{(\bar{f}(|x|), \infty)}(t)}{|x|^{n}} .
$$

Then

$$
\begin{aligned}
\left|\left\{x: g_{t}(x)>s\right\}\right| & =\left|\left\{x: 1 / s>\lambda_{\bar{f}}^{n}(t)+|x|^{n}\right\}\right| \\
& = \begin{cases}0, & \lambda_{\bar{f}}^{n}(t) \geq 1 / s, \\
v_{n}\left(1 / s-\lambda_{\bar{f}}^{n}(t),\right. & \lambda_{\bar{f}}^{n}(t)<1 / s .\end{cases}
\end{aligned}
$$

Similarly,

$$
\begin{aligned}
\left|\left\{x: h_{t}(x)>s\right\}\right| & =\left|\left\{x: \bar{f}(|x|)<t, 1 /|x|^{n}>s\right\}\right| \\
& =\left|\left\{x: \lambda_{\bar{f}}(t) \leq|x|<1 / s^{1 / n}\right\}\right| \\
& = \begin{cases}0, & \lambda_{\bar{f}}^{n}(t) \geq 1 / s, \\
v_{n}\left(1 / s-\lambda_{\bar{f}}^{n}(t)\right), & \lambda_{\bar{f}}^{n}(t)<1 / s .\end{cases}
\end{aligned}
$$


Therefore, $g_{t}$ and $h_{t}$ are equimeasurable functions, and hence

$$
\left\|\frac{\chi_{(\bar{f}(|\cdot|), \infty)}(t)}{|\cdot|^{n}}\right\|_{X}=\left\|\frac{1}{\lambda_{\bar{f}}^{n}(t)+|\cdot|^{n}}\right\|_{X},
$$

which proves (5). The optimality of the inequality follows by choosing $f(x)=$ $\chi_{B}(x)=\chi_{[0,1)}(|x|)$ :

$$
S_{n} f(x)-f(x)= \begin{cases}0, & |x|<1, \\ 1 /|x|^{n}, & |x| \geq 1,\end{cases}
$$

and, as we did in (6), we get

$$
\int_{0}^{\infty} \lambda_{\bar{f}}^{n}(t)\left\|\frac{1}{\lambda_{\bar{f}}^{n}(t)+|\cdot|^{n}}\right\|_{X} d t=\left\|\frac{1}{1+|\cdot|^{n}}\right\|_{X}=\left\|S_{n} f-f\right\|_{X} .
$$

Theorem 5 motivates the study of the class of functions for which the right hand side of the inequality (5) is finite.

Definition 6. Let $X$ be an r.i. space in $\mathbb{R}^{n}$. We define the class of functions

$$
R(X)=\left\{f \in \mathcal{M}\left(\mathbb{R}^{n}\right):\|f\|_{R(X)}<\infty\right\},
$$

where

$$
\|f\|_{R(X)}=v_{n}^{-1} \int_{0}^{\infty} \lambda_{f}(t)\left\|\frac{1}{v_{n}^{-1} \lambda_{f}(t)+|\cdot|^{n}}\right\|_{X} d t<\infty .
$$

REMARK 7.

(i) Using (3) we see that if $f \in X_{\text {rd }}$, then

$$
\|f\|_{R(X)}=\int_{0}^{\infty} \lambda_{\bar{f}}^{n}(t)\left\|\frac{1}{\lambda_{\bar{f}}^{n}(t)+|\cdot|^{n}}\right\|_{X} d t .
$$

(ii) It is easy to see that $\|f\|_{R(X)}=0$ if and only if $f \equiv 0$. Moreover, $\|a f\|_{R(X)}=|a|\|f\|_{R(X)}$.

(iii) A necessary condition for $R(X) \neq\{0\}$ is that there exists a $\delta>0$ such that $1 /\left(|x|^{n}+\delta\right) \in X$, which is equivalent to $L^{1, \infty} \cap L^{\infty} \subset X$. In particular, $R\left(L^{1}\right)=\{0\}$.

(iv) Theorem 5 can now be rewritten as $\left\|S_{n} f-f\right\|_{X} \leq\|f\|_{R(X)}$ for $f \in X_{\text {rd }}$.

We are now going to identify $R(X)$ for some particular cases, like the Lorentz space $L^{p, q}\left(\mathbb{R}^{n}\right)$, with exact norms. In Theorem 13 we will prove a more general result for an r.i. space $X$, but only up to equivalence of norms. We consider first the definition of $L^{p, q}\left(\mathbb{R}^{n}\right)$ in terms of the standard norm, 
which is only valid in the range $1 \leq p<\infty, 1 \leq q \leq p$ :

$$
\|f\|_{p, q}=p^{1 / q}\left(\int_{0}^{\infty}\left(t \lambda_{f}^{1 / p}(t)\right)^{q} \frac{d t}{t}\right)^{1 / q}=\left(\int_{0}^{\infty}\left(t^{1 / p} f^{*}(t)\right)^{q} \frac{d t}{t}\right)^{1 / q},
$$

where $f^{*}$ is the nonincreasing rearrangement of $f[3]$.

Proposition 8. If $1<p<\infty, 1 \leq q \leq p$, and $L^{p, q}\left(\mathbb{R}^{n}\right)$ is endowed with the norm $\|\cdot\|_{p, q}$, then

$$
\|f\|_{R\left(L^{p, q}\right)}=p^{-1 / q^{\prime}}\left(\frac{\Gamma\left(\frac{(p-1) q}{p}\right) \Gamma\left(\frac{p+q}{p}\right)}{\Gamma(q+1)}\right)^{1 / q}\|f\|_{p, 1} .
$$

Proof. Fix $a>0$ and consider $\varphi_{a}(x)=1 /\left(a+|x|^{n}\right)$. Then, as in Theorem 5 ,

$$
\begin{aligned}
\left\|\varphi_{a}\right\|_{p, q} & =p^{1 / q}\left(\int_{0}^{1 / a} v_{n}^{q / p}\left(\frac{1}{t}-a\right)^{q / p} t^{q-1} d t\right)^{1 / q} \\
& =p^{1 / q} v_{n}^{1 / p}\left(\int_{a}^{\infty}(u-a)^{q / p} u^{-q-1} d u\right)^{1 / q} \\
& =p^{1 / q} v_{n}^{1 / p}\left(\frac{\Gamma\left(\frac{(p-1) q}{p}\right) \Gamma\left(\frac{p+q}{p}\right)}{\Gamma(q+1)}\right)^{1 / q} a^{1 / p-1} .
\end{aligned}
$$

Therefore,

$$
\begin{aligned}
\|f\|_{R\left(L^{p, q}\right)} & =v_{n}^{-1} \int_{0}^{\infty} \lambda_{f}(t)\left\|\varphi_{v_{n}^{-1} \lambda_{f}(t)}\right\|_{p, q} d t \\
& =\frac{p^{1 / q}}{v_{n}^{1-1 / p}} \int_{0}^{\infty} \lambda_{f}(t)\left(\frac{\Gamma\left(\frac{(p-1) q}{p}\right) \Gamma\left(\frac{p+q}{p}\right)}{\Gamma(q+1)}\right)^{1 / q}\left(\frac{\lambda_{f}(t)}{v_{n}}\right)^{1 / p-1} d t \\
& =p^{-1 / q^{\prime}}\left(\frac{\Gamma\left(\frac{(p-1) q}{p}\right) \Gamma\left(\frac{p+q}{p}\right)}{\Gamma(q+1)}\right)^{1 / q}\|f\|_{p, 1} \text {. }
\end{aligned}
$$

As a consequence of this result, we can find the best constant for the boundedness of $S_{n}-\mathrm{Id}$, for radially decreasing functions on $L^{p, 1}\left(\mathbb{R}^{n}\right)$.

Proposition 9. If $1<p<\infty, 1 \leq q \leq p$, and $L^{p, q}\left(\mathbb{R}^{n}\right)$ is endowed with the norm $\|\cdot\|_{p, q}$, then for a radially decreasing function $f \in L^{p, 1}\left(\mathbb{R}^{n}\right)$,

$$
\left\|S_{n} f-f\right\|_{p, q} \leq p^{-1 / q^{\prime}}\left(\frac{\Gamma\left(\frac{(p-1) q}{p}\right) \Gamma\left(\frac{p+q}{p}\right)}{\Gamma(q+1)}\right)^{1 / q}\|f\|_{p, 1},
$$

and the inequality is sharp. In particular: 
(i) if $q=p$ and $f \in L_{\mathrm{rd}}^{p, 1}\left(\mathbb{R}^{n}\right)$, then

$$
\left\|S_{n} f-f\right\|_{p} \leq \frac{1}{p(p-1)^{1 / p}}\|f\|_{p, 1}
$$

(ii) if $q=1$ and $f \in L_{\mathrm{rd}}^{p, 1}\left(\mathbb{R}^{n}\right)$, then

$$
\left\|S_{n} f-f\right\|_{p, 1} \leq \frac{\pi}{p \sin (\pi / p)}\|f\|_{p, 1},
$$

and the inequalities are sharp.

Proof. This follows easily using Theorem 5, Remark 7(iv) and Proposition 8, and estimating the constant in (7) for the particular cases.

REMARK 10. (i) It is important to observe that (7) does not follow from the case $q=1$ and the embedding $L^{p, 1} \subset L^{p, q}$. In fact, it is known (see, e.g., [8]) that $\|f\|_{p, q} \leq \frac{1}{p}\left(\frac{p}{q}\right)^{1 / q}\|f\|_{p, 1}$ (with optimal bound). But, if $1<q \leq p$, then

$$
p^{-1 / q^{\prime}}\left(\frac{\Gamma\left(\frac{(p-1) q}{p}\right) \Gamma\left(\frac{p+q}{p}\right)}{\Gamma(q+1)}\right)^{1 / q}<\frac{1}{p}\left(\frac{p}{q}\right)^{1 / q} \frac{\pi}{p \sin (\pi / p)} .
$$

(ii) If $f \in L^{p}\left(\mathbb{R}^{+}\right)$is a decreasing function (this is denoted as $f \in C^{p}$ in $[10])$ and $\widetilde{f}(x)=f(|x|)$, then $\|S f-f\|_{L^{p}\left(\mathbb{R}^{+}\right)}=2^{-1 / p}\left\|S_{1} \widetilde{f}-\widetilde{f}\right\|_{L^{p}(\mathbb{R})}$ and $\|f\|_{L^{p}\left(\mathbb{R}^{+}\right)}=2^{-1 / p}\|\widetilde{f}\|_{L^{p}(\mathbb{R})}$. Using now Proposition $9(\mathrm{i})$, we can prove the optimal restricted type estimate (for $f \in C^{p}$ and $p>1$ )

$$
\|S f-f\|_{L^{p}\left(\mathbb{R}^{+}\right)} \leq \frac{1}{p(p-1)^{1 / p}}\|f\|_{L^{p, 1}\left(\mathbb{R}^{+}\right)} .
$$

As before, using Proposition 9(ii), we find that for a decreasing function $f \in L^{p, 1}\left(\mathbb{R}^{+}\right)$and $p>1$,

$$
\|S f-f\|_{L^{p, 1}\left(\mathbb{R}^{+}\right)} \leq \frac{\pi}{p \sin (\pi / p)}\|f\|_{L^{p, 1}\left(\mathbb{R}^{+}\right)} .
$$

These results should be compared with the strong type inequality proved in [10], under the hypothesis that $f \in C^{p}$ and $p \in\{2,3, \ldots\}$,

$$
\|S f-f\|_{L^{p}\left(\mathbb{R}^{+}\right)} \leq \frac{1}{(p-1)^{1 / p}}\|f\|_{L^{p}\left(\mathbb{R}^{+}\right)} .
$$

(iii) For the case $1<p<q \leq \infty$ we can also obtain a result like (7), although this time we cannot guarantee its sharpness. The main observation is that, for this range of indices, $\|\cdot\|_{p, q}$ is a quasinorm for which the best constant in Minkowski's integral inequality is (see [2])

$$
\left\|\int F(\cdot, y) d y\right\|_{p, q} \leq\left(\frac{p}{q}\right)^{1 / q}\left(\frac{p^{\prime}}{q^{\prime}}\right)^{1 / q^{\prime}} \int\|F(\cdot, y)\|_{p, q} d y
$$


Therefore, as in Theorem 5 and observing that the proof of Proposition 8 also works if $1<p<q \leq \infty$ (the case $q=\infty$ with the constant $1 /\left(p p^{\prime}(p-1)^{1 / p}\right)$ ), we get, for $f \in L_{\mathrm{rd}}^{p, 1}\left(\mathbb{R}^{n}\right)$,

$$
\left\|S_{n} f-f\right\|_{p, q} \leq\left(\frac{p}{q}\right)^{1 / q}\left(\frac{p^{\prime}}{q^{\prime}}\right)^{1 / q^{\prime}} p^{-1 / q^{\prime}}\left(\frac{\Gamma\left(\frac{(p-1) q}{p}\right) \Gamma\left(\frac{p+q}{p}\right)}{\Gamma(q+1)}\right)^{1 / q}\|f\|_{p, 1}
$$

if $1<p<q<\infty$, and

$$
\left\|S_{n} f-f\right\|_{p, \infty} \leq \frac{1}{p(p-1)^{1 / p}}\|f\|_{p, 1} .
$$

Similarly to what we have done for the norm $\|\cdot\|_{p, q}, 1<p<\infty, 1 \leq$ $q \leq p$, we now consider the usual renorming of the $L^{p, q}\left(\mathbb{R}^{n}\right)$ spaces, for the whole range of indices $1<p<\infty, 1 \leq q \leq \infty$, in terms of the maximal norm (see [13]):

$$
\|f\|_{p, q}^{*}=\left(\int_{0}^{\infty}\left(t^{1 / p} f^{* *}(t)\right)^{q} \frac{d t}{t}\right)^{1 / q},
$$

where $f^{* *}(t)$ is the maximal function of $f$ (see Remark 2). We will denote by $L_{*}^{p, q}\left(\mathbb{R}^{n}\right)$ the space $L^{p, q}\left(\mathbb{R}^{n}\right)$ endowed with the norm $\|\cdot\|_{p, q}^{*}$.

Proposition 11. If $1<p<\infty, 1 \leq q \leq \infty$, and $f \in L_{*}^{p, q}\left(\mathbb{R}^{n}\right)$, then

$$
\|f\|_{R\left(L_{*}^{p, q}\right)}=\frac{1}{p p^{\prime}}\left(\int_{0}^{\infty} \log ^{q}(1+s) s^{-q / p^{\prime}-1} d s\right)^{1 / q}\|f\|_{p, 1}^{*}
$$

if $1 \leq q<\infty$, and

$$
\|f\|_{R\left(L_{*}^{p, \infty}\right)}=\frac{(p-1)^{1 / p^{\prime}}}{p p^{\prime}} \log \left(p^{\prime}\right)\|f\|_{p, 1}^{*} .
$$

Proof. As in Proposition 8, fix $a>0$ and consider $\varphi_{a}(x)=1 /\left(a+|x|^{n}\right)$. Then

$$
\varphi_{a}^{* *}(t)=\frac{v_{n}}{t} \log \left(1+\frac{t}{v_{n} a}\right) .
$$

If $1 \leq q<\infty$,

$$
\left\|\varphi_{a}\right\|_{p, q}^{*}=a^{-1 / p^{\prime}} v_{n}^{1 / p}\left(\int_{0}^{\infty} \log ^{q}(1+s) s^{-q / p^{\prime}-1} d s\right)^{1 / q},
$$

and hence, using that $p^{\prime}\|f\|_{p, 1}=\|f\|_{p, 1}^{*}$ (see, e.g., [2]),

$$
\begin{aligned}
\|f\|_{R\left(L_{*}^{p, q}\right)} & =\int_{0}^{\infty} \lambda_{f}(t)^{1 / p}\left(\int_{0}^{\infty} \log ^{q}(1+s) s^{-q / p^{\prime}-1} d s\right)^{1 / q} d t \\
& =\frac{1}{p p^{\prime}}\left(\int_{0}^{\infty} \log ^{q}(1+s) s^{-q / p^{\prime}-1} d s\right)^{1 / q}\|f\|_{p, 1}^{*} .
\end{aligned}
$$


If $q=\infty$, then

$$
\left\|\varphi_{a}\right\|_{p, \infty}^{*}=v_{n}(p-1)^{1 / p^{\prime}} \log \left(p^{\prime}\right)\left(v_{n} a\right)^{-1 / p^{\prime}},
$$

and

$$
\begin{aligned}
\|f\|_{R\left(L_{*}^{p, \infty}\right)} & =v_{n}^{-1} \int_{0}^{\infty} \lambda_{f}(t) v_{n}(p-1)^{1 / p^{\prime}} \log \left(p^{\prime}\right)\left(v_{n} \frac{\lambda_{f}(t)}{v_{n}}\right)^{-1 / p^{\prime}} d t \\
& =\frac{(p-1)^{1 / p^{\prime}}}{p} \log p^{\prime}\|f\|_{p, 1}=\frac{(p-1)^{1 / p^{\prime}}}{p p^{\prime}} \log \left(p^{\prime}\right)\|f\|_{p, 1}^{*} .
\end{aligned}
$$

Proposition 12. Let $1<p<\infty, 1 \leq q \leq \infty$, and $f \in L_{*}^{p, q}\left(\mathbb{R}^{n}\right)$ be a radially decreasing function. Then

$$
\left\|S_{n} f-f\right\|_{p, q}^{*} \leq \frac{1}{p p^{\prime}}\left(\int_{0}^{\infty} \log ^{q}(1+s) s^{-q / p^{\prime}-1} d s\right)^{1 / q}\|f\|_{p, 1}^{*}
$$

if $1 \leq q<\infty$, and

$$
\left\|S_{n} f-f\right\|_{p, \infty}^{*} \leq \frac{(p-1)^{1 / p^{\prime}}}{p p^{\prime}} \log \left(p^{\prime}\right)\|f\|_{p, 1}^{*},
$$

and both inequalities are sharp. In particular:

(i) if $q=p^{\prime}$ and $f \in L_{*, \mathrm{rd}}^{p, p^{\prime}}\left(\mathbb{R}^{n}\right)$, then

$$
\left\|S_{n} f-f\right\|_{p, p^{\prime}}^{*} \leq \frac{\left(\Gamma\left(p^{\prime}+1\right) \zeta\left(p^{\prime}\right)\right)^{1 / p^{\prime}}}{p p^{\prime}}\|f\|_{p, 1}^{*} ;
$$

(ii) if $q=1$ and $f \in L_{*, \mathrm{rd}}^{p, 1}\left(\mathbb{R}^{n}\right)$, then

$$
\left\|S_{n} f-f\right\|_{p, 1}^{*} \leq \frac{\pi}{p \sin (\pi / p)}\|f\|_{p, 1}^{*}
$$

and the inequalities are sharp.

Proof. This follows easily using Theorem 5 , Remark 7 (iv) and Proposition 11, and estimating the constant in (8) for the particular cases.

Even though we have shown that $R\left(L^{1}\right)=\{0\}$, we see that, in many other cases, $R(X)$ is isometric, up to a multiplicative constant, to the minimal Lorentz space $\Lambda(X)$. This is the case for $X=L^{p, q}$ and $X=L_{*}^{p, q}$ (see Propositions 8 and 11) for which the minimal space corresponds to the in$\operatorname{dex} q=1$ (see [3]). We will now study this observation for a general $X$, and show that, under some weak assumptions, $R(X)$ and $\Lambda(X)$ coincide. Recall that

$$
\Lambda(X)=\left\{f \in \mathcal{M}\left(\mathbb{R}^{n}\right): \int_{0}^{\infty} f^{*}(t) d \varphi_{X}(t)<\infty\right\},
$$

where $\varphi_{X}(t)=\left\|\chi_{E}\right\|_{X}(|E|=t)$ is the fundamental function of $X$. To simplify the calculations we are going to assume that $\lim _{t \rightarrow 0^{+}} \varphi_{X}(t)=0$ (see 
[3. Chapter 2, Theorem 5.5] for a characterization of this property), which is always true for the $L^{p, q}$ spaces (observe that we exclude the nonseparable case of $\left.L^{\infty}\right)$. Then we can write

$$
\|f\|_{\Lambda(X)}=\int_{0}^{\infty} f^{*}(t) \varphi_{X}^{\prime}(t) d t=\int_{0}^{\infty} \varphi_{X}\left(\lambda_{f}(t)\right) d t .
$$

Using [3, Chapter 2, Proposition 5.11] we can assume, without loss of generality, that $\varphi_{X}$ is a (nonnegative and nondecreasing) concave function, so that $\|\cdot\|_{\Lambda(X)}$ is a norm. We will also make use of the fact that

$$
D_{X} \equiv \sup _{t>0} \frac{\varphi_{X}(2 t)}{\varphi_{X}(t)} \in[1,2]
$$

(this is known as the $\Delta_{2}$-condition): fix $t>0$ and choose two disjoint sets with $|A|=|B|=t$. Then

$$
\varphi_{X}(2 t)=\left\|\chi_{A \cup B}\right\|_{X}=\left\|\chi_{A}+\chi_{B}\right\|_{X} \leq\left\|\chi_{A}\right\|_{X}+\left\|\chi_{B}\right\|_{X}=2 \varphi_{X}(t) .
$$

For example, if $X=\left(L^{p, q}\left(\mathbb{R}^{n}\right),\|\cdot\|_{p, q}\right)(1 \leq q \leq p<\infty)$ or $X=\left(L^{p, q}\left(\mathbb{R}^{n}\right),\|\cdot\|_{p, q}^{*}\right)$ $(1<p<\infty, 1 \leq q \leq \infty$ or $p=q=1)$, then $D_{X}=2^{1 / p}$.

TheOREM 13. Let $X$ be an r.i. space.

(i) For every $f \in R(X)$,

$$
\|f\|_{\Lambda(X)} \leq 2\|f\|_{R(X)} .
$$

(ii) If $D_{X} \in[1,2)$ (see (10)), then for every $f \in \Lambda(X)$,

$$
\|f\|_{R(X)} \leq \frac{2}{2-D_{X}}\|f\|_{\Lambda(X)} .
$$

In particular, if $D_{X} \in[1,2)$ then $R(X)=\Lambda(X)$.

Proof. To prove (i), we consider the ball $B_{t}=B\left(0,\left(v_{n}^{-1} t\right)^{1 / n}\right)$, so that $\left|B_{t}\right|=t$. Then $\chi_{B_{t}}(x) \leq 2 t /\left(t+v_{n}|x|^{n}\right)$ and hence

$$
\varphi_{X}(t) \leq 2 v_{n}^{-1} t\left\|\frac{1}{v_{n}^{-1} t+|\cdot|^{n}}\right\|_{X} .
$$

Therefore,

$$
\begin{aligned}
\|f\|_{\Lambda(X)} & =\int_{0}^{\infty} \varphi_{X}\left(\lambda_{f}(t)\right) d t \\
& \leq 2 v_{n}^{-1} \int_{0}^{\infty} \lambda_{f}(t)\left\|\frac{1}{v_{n}^{-1} \lambda_{f}(t)+|\cdot|^{n}}\right\|_{X} d t=2\|f\|_{R(X)} .
\end{aligned}
$$


(ii) If we now assume that $D_{X} \in[1,2)$, set $S_{r}=B_{2 r} \backslash B_{r}$. Then

$$
\begin{aligned}
\frac{t}{t+v_{n}|x|^{n}} & =\sum_{k=1}^{\infty} \frac{t}{t+v_{n}|x|^{n}} \chi_{S_{2^{k}}}(x)+\frac{t}{t+v_{n}|x|^{n}} \chi_{B_{t}}(x) \\
& \leq \sum_{k=1}^{\infty} \frac{1}{1+2^{k}} \chi_{S_{2^{k}}}(x)+\chi_{B_{t}}(x) .
\end{aligned}
$$

Thus,

$$
\begin{aligned}
\left\|\frac{t}{t+v_{n}|\cdot|^{n}}\right\|_{X} & \leq \sum_{k=1}^{\infty} \frac{1}{1+2^{k}}\left\|\chi_{S_{2^{k}}}\right\|_{X}+\left\|\chi_{B_{t}}\right\|_{X} \\
& =\sum_{k=1}^{\infty} \frac{1}{1+2^{k}} \varphi_{X}\left(2^{k} t\right)+\varphi_{X}(t) \\
& \leq \sum_{k=0}^{\infty} \frac{D_{X}^{k}}{2^{k}} \varphi_{X}(t)=\frac{2}{2-D_{X}} \varphi_{X}(t) .
\end{aligned}
$$

Hence,

REMARK 14.

$$
\|f\|_{R(X)} \leq \frac{2}{2-D_{X}}\|f\|_{\Lambda(X)}
$$

(i) As already observed in Remark 7, $R\left(L^{1}\right)=\{0\} \neq \Lambda\left(L^{1}\right)=L^{1}$, and hence the embedding $\Lambda(X) \subset R(X)$ of Theorem 13 (ii) is not true, in general, for the case $D_{X}=2$.

(ii) We have seen in Propositions 8 and 11 that, for the $L^{p, q}$ spaces, $\|\cdot\|_{R(X)}$ is a multiple of $\|\cdot\|_{\Lambda(X)}$. However, this is not true in general. We recall the definition of the weighted Lorentz space:

$$
\Lambda^{p}(w)=\left\{f:\|f\|_{\Lambda^{p}(w)}=\left(\int_{0}^{\infty}\left(f^{*}(t)\right)^{p} w(t) d t\right)^{1 / p}<\infty\right\},
$$

and consider $X=\Lambda^{2}(w), w=\chi_{(0,1)}$, and $f_{r}^{*}(t)=\chi_{(0, r)}(t)$. Then

$$
\left\|f_{r}\right\|_{\Lambda(X)}= \begin{cases}\sqrt{r} & \text { if } r<1, \\ 1 & \text { if } r>1,\end{cases}
$$

but

$$
\left\|f_{r}\right\|_{R(X)}=\sqrt{\frac{r}{1+r}} .
$$

(iii) If $w$ is a decreasing weight (so that $\|\cdot\|_{\Lambda^{p}(w)}$ is a norm [11]), then $D_{\Lambda^{p}(w)} \leq 2^{1 / p}$, and hence $D_{\Lambda^{p}(w)}<2$, whenever $p>1$. Therefore, Theorem 13 applies. More general results can be obtained for weights in the $B_{p}$ class (see $[1,7]$ ). 
Acknowledgments. This research was partially supported by grants MTM2007-60500 and 2005SGR00556.

\section{References}

[1] M. A. Ariño and B. Muckenhoupt, Maximal functions on classical Lorentz spaces and Hardy's inequality with weights for nonincreasing functions, Trans. Amer. Math. Soc. 320 (1990), 727-735.

[2] S. Barza, V. Kolyada, and J. Soria, Sharp constants related to the triangle inequality in Lorentz spaces, ibid. 361 (2009), 5555-5574.

[3] C. Bennett and R. Sharpley, Interpolation of Operators, Academic Press, 1988.

[4] S. Boza and J. Martín, Equivalent expressions for norms in classical Lorentz spaces, Forum Math. 17 (2005), 361-373.

[5] M. J. Carro, A. Gogatishvili, J. Martín, and L. Pick, Functional properties of rearrangement invariant spaces defined in terms of oscillations, J. Funct. Anal. 229 (2005), 375-404.

[6] M. J. Carro, L. Pick, J. Soria, and V. D. Stepanov, On embeddings between classical Lorentz spaces, Math. Inequal. Appl. 4 (2001), 397-428.

[7] M. J. Carro, J. A. Raposo, and J. Soria, Recent developments in the theory of Lorentz spaces and weighted inequalities, Mem. Amer. Math. Soc. 187 (2007), no. 877.

[8] M. J. Carro and J. Soria, Weighted Lorentz spaces and the Hardy operator, J. Funct. Anal. 112 (1993), 480-494.

[9] M. Christ and L. Grafakos, Best constants for two nonconvolution inequalities, Proc. Amer. Math. Soc. 123 (1995), 1687-1693.

[10] N. Kruglyak and E. Setterqvist, Sharp estimates for the identity minus Hardy operator on the cone of decreasing functions, ibid. 136 (2008), 2505-2513.

[11] G. G. Lorentz, Some new functional spaces, Ann. of Math. 51 (1950), 37-55.

[12] E. Sawyer, Boundedness of classical operators on classical Lorentz spaces, Studia Math. 96 (1990), 145-158.

[13] E. M. Stein and G. Weiss, Introduction to Fourier Analysis on Euclidean Spaces, Princeton Univ. Press, 1971.

Javier Soria

Department of Applied Mathematics and Analysis

University of Barcelona

E-08007 Barcelona, Spain

E-mail: soria@ub.edu 